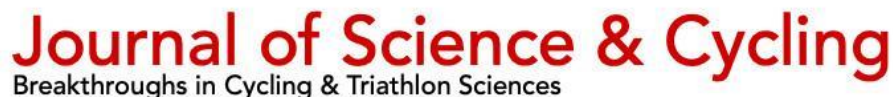

\section{Does participation in Downhill mountain biking affect measures of executive function?}

\author{
Howard Thomas Hurst ${ }^{1}$, Stuart Hancock ${ }^{2}$, Jack Hardwicke ${ }^{3}$, Eric Anderson ${ }^{4}$ \\ 1 University of Central Lancashire \\ 2 School for Sport and Wellbeing, University of Central Lancashire, Preston, UK \\ ${ }^{3}$ Department of Sport, Exercise and Health, University of Winchester, Winchester, UK \\ ${ }^{4}$ Department of Sport, Exercise and Health, University of Winchester, Winchester, UK
}

* Correspondence: Howard Thomas Hurst. hthurst@uclan.ac.uk

Received: 7 September 2020; Accepted: 8 September 2020; Published: 13 October 2020

\begin{abstract}
This study assessed the influence of downhill mountain biking on acute measures of executive function. Twenty-three participants took part in the study and were assigned to either, FIELD ( $\mathrm{N}=7$, mean age $27 \pm 9 \mathrm{yrs}), \mathrm{LAB}(\mathrm{N}=8$, mean age $36 \pm 9 \mathrm{yrs})$ or CONTROL $(\mathrm{N}=8$, mean age 41 \pm 9 yrs) groups. Participants performed the Stroop colour-word test via a tablet pc app (EncephalApp). Additionally, the Trail Making Test A (TMT-A) and B (TMT-B) were completed. Significant main effects were found for OffTime $\left(\mathrm{F}_{2,12}=13.04 ; \mathrm{p}=.001 ; \eta_{\mathrm{p}}{ }^{2}=.69\right)$, OnTime $\left(\mathrm{F}_{2,12}=4.31\right.$; $\left.\mathrm{p}=.04 ; \eta_{\mathrm{p}}{ }^{2}=.42\right)$ and OnTrials $\left(\mathrm{F}_{2,12}=10.74 ; \mathrm{p}=.002 ; \eta_{\mathrm{p}}{ }^{2}=.64\right)$, with the results showing a decrement in Stroop test performance following Trial1 and Trial4 compared to BL for the FIELD group. A significant main effect was found for TMT-A $\left(\mathrm{F}_{2,12}=7.50 ; \mathrm{p}=.008 ; \eta_{\mathrm{p}}{ }^{2}=.56\right)$, with performance improving following Trial4 compared to BL for the FIELD group. LAB and CONTROL groups significantly improved on the TMT-B from BL to Trial1 and Trial4 $\left(\mathrm{F}_{2,14}=4.31 ; \mathrm{p}=.04 ; \eta_{\mathrm{p}}{ }^{2}=.38\right.$ and $\mathrm{F}_{2,14}=13.70 ; \mathrm{p}=.001 ; \eta_{\mathrm{p}}^{2}=.66$, respectively). Results suggest participation in downhill mountain biking in this cohort significantly impaired executive function when compared to repeated sprint activity within a laboratory. This is indicative of repeated head accelerations caused by riding over rough terrain.
\end{abstract}

Keywords: mountain biking; executive function; Chronic Traumatic Encephalopathy; head injuries.

\section{Introduction}

The past decade has seen an increase in research focused on the diagnosis and management of sports related head injuries, commonly described within sport as concussion, but medically understood to be a Traumatic Brain Injury (TBI) or mild Traumatic Brain Injury (mTBI). It is important to clarify that concussion is just one of a number of brain injuries that fall under the umbrella of mTBI. These injuries result from impacts or jolts to the head or body, resulting in transmitted forces causing an impact between the brain and the skull (Meaney \& Smith, 2011; Stern \& Riley, 2011). Current research has focused primarily on the prevalence of, and diagnostic criteria concerning TBI and mTBI among contact sports, such as American football, soccer, rugby and hockey (Guskiewicz et al., 2003; 
Broglio et al., 2011; Donaldson, Ashbridge \& Cusimani, 2013; Gardner et al., 2015). However, other non-contact sports, such as BMX and Mountain biking (MTB), may also put competitors at risk of sustaining a TBI or mTBI (Kronisch, Pfeiffer \& Chow, 1996; Becker et al., 2013; Hurst et al., 2018). Whilst the greatest risk of brain injury in these sports comes from crashing, riders may be at risk simply from riding over rough terrain, as the brain is subject to repeated translational and rotational accelerations (Hurst, Atkins \& Dickinson, 2018).

Downhill MTB (DHI) in particular, is characterised by fast, open mountain tracks, and technical woodland sections. It requires participants to negotiate jumps, rocks, roots and vertical drops. Subsequently, the risk of crashing and sustaining a head injury is elevated. Becker et al. (2013), reported that symptomatic concussions accounted for $5 \%$ of accidents during DHI. However, like for other sports, the true number of cases of mTBI may be much higher in DHI then previously reported, as under reporting of head injuries is common across sports (Kroshus et al., 2015).

Although there is no agreed-upon impactdose for producing symptoms of concussion, Guskiewicz et al. (2007) found symptoms can appear at $60 \mathrm{~g}$. Studied among DHI athletes, Hurst, Atkins, and Dickinson (2018), reported mean and peak translational head accelerations of $24.5 \mathrm{~g}$ and $79.9 \mathrm{~g}$ respectively. Similarly, rotational acceleration of 5,500 $\mathrm{rad} / \mathrm{s}^{2}$ have been associated with concussion in American football players (Broglio et al., 2010), whilst DHI athletes reported mean and peak rotational accelerations of $2621.2 \mathrm{rad} / \mathrm{s}^{2}$ and $8566.8 \mathrm{rad} / \mathrm{s}^{2}$, respectively (Hurst, Atkins \& Dickinson, 2018). Such head accelerations therefore strongly indicate an elevated risk of sustaining a TBI or mTBI within DHI.

In addition to acute brain injuries, repeated sub-concussive impacts have been linked to neurodegenerative conditions, including Alzheimer's (Russell et al., 2019) and Chronic Traumatic Encephalopathy (CTE), in collision sport athletes (Maroon et al., 2015). These accelerations are termed 'subconcussive' because they are not identifiable by concussion tests, but may nonetheless result in cerebral damage and injury to the central nervous system (Broglio et al., 2011; Spiotta et al., 2011). Alosco et al. (2018), suggests that, among American football players, a threshold dose for CTE appears to be 4.5 or more years of play, at which point athletes will have sustained thousands of sub-concussive impacts (Mez et al., 2020).

Relating CTE findings to DHI, less is understood about the long-term effect of repeated head accelerations resulting from non-crashing trail vibrations. However, an association of at least temporary and intermediate neurological functioning may be comparable to studies of participants heading a soccer ball. Di Virgilio et al. (2016), reported that heading a soccer ball 20 times in ten minutes resulted in transient, but nonetheless impairment, of short and longterm memory function. Similarly, McAllister et al. (2014), found that repeated head accelerations of a sub-concussive magnitude impaired cognition and white matter integrity in contact sport athletes.

Whether this cognitive diminishment is short or long term is not known; but given competitive athletes normally play for repeated seasons, it raises concerns about long-term cognitive health. Given the demanding terrain encountered during DHI, and the competitive nature of the sport which sees many athletes competing for consecutive years, it is plausible that similar repeated head accelerations resulting from trail vibrations may also negatively affect cognition in DHI competitors.

Whilst the health-related fitness benefits of DHI have previously been reported (Burr et al., 2012), no data currently exists for the sport pertaining to brain health, despite emerging evidence for the detrimental effects of these repeated low magnitude head accelerations in contact sports. Therefore, the aim of this study was to investigate the possible effect multiple DHI runs over a single day have on executive function. It was hypothesised that measures of executive function would be impaired. 


\section{Materials and Methods}

\section{Participants}

Twenty-three participants took part in the study, comprising three groups, determined by purposeful sampling. FIELD $(\mathrm{N}=7$, mean age $27 \pm 9 \mathrm{yrs}), \mathrm{LAB}(\mathrm{N}=8$, mean age $36 \pm 9$ yrs) and CONTROL $(\mathrm{N}=8$, mean age $41 \pm 9$ yrs). The FIELD group was made up of male Elite DHI mountain bikers with a minimum of four years racing experience at National and International competition. The sample size of the FIELD group represented $12 \%$ of all British Cycling registered Elite male DHI riders for the 2018 season. Additionally, all had previous experience of riding the chosen test course. The LAB group was a mix of males and females who regularly participated in gym-based cycling spin classes for at least 12 months, whilst the CONTROL group was composed of male and female sport science students for which no cycling proficiency was required. Written and informed consent was acquired prior to testing and the study was granted ethical approval by the University of Central Lancashire STEMH ethics committee (reference: STEMH 718) and in accordance with the Declaration of Helsinki 1975, revised 2013.

\section{Experimental design}

A Stroop colour-word test was used to determine changes in executive function, using the previously validated tablet pc application (app) EncephalApp_Stroop (Bajaj et al., 2015). In order to perform well, DHI riders are required to brake as little as possible in order to maintain velocity. Additionally, heavy braking in response to unexpected events on such technical courses may increase the risk of crashing. However, for most individuals the automatic response when faced with approaching obstacles in order to avoid crashing is to brake (McGehee \& Carsten, 2010), yet such behavior might be counterintuitive in DHI. Given this requirement to frequently inhibit such automatic behavior in DHI and the Stroop tests ability to assess such inhibition, it was deemed appropriate for use in the current study. The app comprised an easier congruent "Off" state and a harder incongruent "On" state. In the Off state, participants were presented with coloured "\#" signs in green, red or blue and were required to identify the colour as quickly as possible. In the On state, participants were presented with incongruent stimuli; e.g. the word "red" printed in green font, for which they had to identify the colour of the font, rather than the word. Within each state, participants were presented with 10 stimuli. Recorded variables were; time to complete five correct Off trials (OffTime), time to complete five correct On trials (OnTime), number of trials required to complete five successive Off state (OffTrials) and five On state (OnTrials) trails without error.

OffTime assessed psychomotor speed, OnTime represented reaction time, whilst the number of trials required reflected processing accuracy. Additionally, OnTimeOffTime was calculated to establish cognitive flexibility. The order of trials to be completed within the app were as follows: (1) 2 practice Off trials (2) 5 correct Off state trials (3) 2 practice On trials and (4) 5 correct On state trials. If participants made an error during any stage the test would re-start from the beginning of that stage. If more than 20 attempts were required for any stage, the app automatically stopped. All times were reported in seconds (s).

Additionally, the Reitan Trail Making Test (Reitan, 1958), part A (TMT-A) and part B (TMT-B) were administered to determine behavioural regulation and motor speed (TMT-A) and mental flexibility (TMT-B). These tests were chosen due to DHI riders frequently being required to shift their focus of attention between trail features immediately in front of them and those further down the track at speed. As such, the TMT tests were deemed suitable. Both parts were administered using pen and paper. TMT-A consisted of a printed sheet of paper with a series of 25 semi-random encircled numbers. Participants were required to trace a line on the paper to connect the numbers in the correct sequence, 1-25, as quickly as 
possible. TMT-B consisted of both 25 numbers and letter in alphabetical order and participants again had to trace a line between them in order, alternating between numbers and letters. For example, the first number " 1 " would be followed by the letter " $\mathrm{A}$ " then number " 2 " and letter " $B$ " and so on. For both parts, if the participant made an error, the tester would direct them back to the last correct number or letter where they would then continue. There were no 'penalties' for errors, though the timer did not stop until the task was complete. A maximum time limit of $300 \mathrm{~s}$ to complete both parts was used as a cut-off to stop the tests (Thompson et al., 1999). Whilst computer/tablet PC versions of the TMT tests were available, the authors found that gloved hands or sweaty bare hands frequently caused problems when tracing their finger over the PC screen. Therefore, the use of traditional paper-based assessments was justified. Unlike, the TMT tests, the Stroop test app was found not to be affected by gloves or damp hands, as participants were simply required to press a button on screen, as opposed to dragging their finger across it. Other computer based programmes, such as ImPACT, were not suitable due to lack of suitable power supply out in the field.

For the FIELD group, data were collected at the Cwmcarn MTB centre, South Wales, UK. The course used was a $1.64 \mathrm{~km}$ purpose built DHI track with a $249 \mathrm{~m}$ vertical drop and was graded as 'extreme'. Participants completed the EncephalApp_Stroop test and Trail Making tests 2 hours prior to riding to establish baseline measures (BL). Whilst the Stroop test had practice built into the app, the Trail Making tests did not. Therefore, participants were allowed 2 practice attempts prior to completing a third trial that would be used as their BL value. The FIELD group then performed four runs of the DHI track, each as quickly as possible, with the cognitive tests being repeated within 5 minutes of completing run 1 (Trial1) and run 4 (Trail4). A total of 28 runs were performed by the FIELD group, with a mean run time of 179.52 $\pm 1.96 \mathrm{~s}$. Riders had a 1-hour passive recovery following their second run, during which they could refuel and hydrate ad libitum. In addition, participants also recovered for approximately 15 minutes between runs during the bus transfer back to the start. All riders rode the same team issue Intense M16 downhill bicycles with identical componentry. Riders bicycles only differed with respect to personal preference for suspension set up.

Participants in the LAB group followed the same protocol timings and were allowed the same number of practice tests and rests as those in the FIELD group. However, rather than field-based cycling, the LAB group performed four $180 \mathrm{~s}$ intermittent repeated sprint tests on a cycle ergometer (Wattbike Pro, Wattbike, UK). The test consisted of sprints of varying duration between 5 and 15 $\mathrm{s}$ with passive recovery period between sprints, again varying in duration. This protocol had previously been used to simulate the stop/start nature of DHI within a laboratory setting and to induce comparable levels of time and fatigue to field-based riding (Hurst \& Atkins, 2006). In the context of the present study, the aim of the laboratory tests was also to simulate field based DHI activity, but without the accompanying head accelerations experienced by the FIELD group and therefore account for the influence of fatigue alone on cognitive function. Cognitive tests were again performed at $\mathrm{BL}$ and after the first (Trial1) and fourth (Trial4) ergometer tests. Finally, those in the CONTROL group again followed the same protocol timings as the other two groups, but performed the cognitive tests only, without any exercise.

\section{Statistical analyses}

Data were analysed using the statistical package SPSS (version 26, IBM Inc., USA). The alpha level was set at $\mathrm{p} \leq 0.05$. A 3 (Group - Between Subjects) x 3 (Time - Within Subjects) mixed methods analysis of variance (ANOVA) was performed to determine any main effects for time and group as well as the presence of an interaction between the two factors. Further planned comparisons using 
univariate and repeated measure ANOVA's were then run to establish where any differences lay between and within groups for each factor. Effect size was calculated using a partial $\operatorname{Eta}^{2}\left(\eta_{\mathrm{p}}{ }^{2}\right)$ and classified as small (0.01), medium (0.09) and large $(>0.25)$ (Cohen, 1988). All data are presented as mean $\pm \mathrm{SD}$.

\section{Results}

No significant main effect was reported between subjects for OffTime $\left(\mathrm{F}_{2,20}=0.12 ; \mathrm{p}=\right.$ $\left.0.89 ; \eta_{\mathrm{p}}{ }^{2}=0.01\right)$. However, a significant time main effect was found within subjects $\left(\mathrm{F}_{2,40}=\right.$ 8.72; $\left.\mathrm{p}=0.001 ; \eta_{\mathrm{p}}{ }^{2}=0.30\right)$, along with a significant interaction main effect $\left(\mathrm{F}_{4,40}=\right.$ 11.68; $\left.\mathrm{p}<0.001 ; \eta_{\mathrm{p}}{ }^{2}=0.54\right)$. Simple main effects were found for the FIELD group $\left(\mathrm{F}_{2,12}\right.$ $\left.=13.04 ; \mathrm{p}=0.001 ; \eta \mathrm{p}^{2}=0.69\right)$, with post-hoc comparisons finding differences between $\mathrm{BL}$ and Trial1 $(\mathrm{p}=0.03)$ and BL and Trial4 $(\mathrm{p}=$ $0.01)$ and the LAB group $\left(F_{2,14}=5.01 ; p=0.02\right.$; $\left.\eta_{\mathrm{p}^{2}}=0.42\right)$, with post-hoc comparisons showing differences between BL and Trial4 $(p=0.01)$. The CONTROL group showed no significant differences in OffTime across the three time points.

No significant main effects were found between subjects for OnTime $\left(\mathrm{F}_{2,20}=0.38 ; \mathrm{p}=\right.$ $\left.0.69 ; \eta_{\mathrm{p}}{ }^{2}=0.04\right)$ or within subjects for time $\left(\mathrm{F}_{2,40}=1.52 ; \mathrm{p}=0.23 ; \eta_{\mathrm{p}}{ }^{2}=0.07\right)$. Significant differences for OnTime were only found within subjects for the interaction main effect $\left(\mathrm{F}_{4,40}=4.07 ; \mathrm{p}<0.007 ; \eta_{\mathrm{p}}{ }^{2}=0.29\right)$. Further simple main effects were found the be significant for the FIELD group $\left(\mathrm{F}_{2,12}=4.31 ; \mathrm{p}\right.$ $\left.=0.04 ; \eta_{\mathrm{p}}{ }^{2}=0.42\right)$, with post-hoc comparisons again revealing differences between $\mathrm{BL}$ and Trial1 $(p=0.01)$ and BL and Trial4 $(p=0.02)$. Neither LAB or CONTROL group showed significant differences in OnTime at BL, Trial1 or Trial4.

No significant differences were revealed for the number of OffTrials attempts either between subjects $\left(\mathrm{F}_{2,20}=0.50 ; \mathrm{p}=0.62 ; \eta_{\mathrm{p}}{ }^{2}=\right.$ $0.05)$ or within subjects for time $\left(\mathrm{F}_{2,40}=0.50 ; \mathrm{p}\right.$ $\left.=0.61 ; \eta_{\mathrm{p}}{ }^{2}=0.03\right)$ and interaction main effects $\left(\mathrm{F}_{4,40}=2.43 ; \mathrm{p}=0.06 ; \eta_{\mathrm{p}}{ }^{2}=0.20\right)$. Similarly, for the OnTrials attempts, no significant main effects were found between subjects $\left(\mathrm{F}_{2,20}=\right.$ $\left.0.34 ; \mathrm{p}=0.72 ; \eta_{\mathrm{p}}{ }^{2}=0.03\right)$. However, there was a within subjects' significant main interaction effect $\left(\mathrm{F}_{2,40}=4.84 ; \mathrm{p}=0.04 ; \eta_{\mathrm{p}}{ }^{2}=0.23\right)$ and a significant within subjects' main effect for time $\left(\mathrm{F}_{2,40}=3.82 ; \mathrm{p}=0.03 ; \eta_{\mathrm{p}}{ }^{2}=0.16\right)$. Simple main effects were found for the FIELD group $\left(\mathrm{F}_{2,12}=10.50 ; \mathrm{p}=0.002 ; \eta_{\mathrm{p}}{ }^{2}=0.64\right)$, with posthoc comparisons showing differences between BL and Trial4 $(\mathrm{p}=0.01)$. No other post-hoc comparisons were significant.

There were no significant main effects found for OnTime-OffTime either between subjects $\left(\mathrm{F}_{2,20}=2.59 ; \mathrm{p}=0.10 ; \eta_{\mathrm{p}}{ }^{2}=0.21\right)$ or within subjects for time $\left(\mathrm{F}_{2,40}=1.65 ; \mathrm{p}=0.20 ; \eta_{\mathrm{p}}{ }^{2}=\right.$ $0.08)$ and interaction main effects $\left(\mathrm{F}_{4,40}=0.44\right.$; $\left.\mathrm{p}=0.78 ; \eta_{\mathrm{p}}{ }^{2}=0.04\right)$. However, when planned comparisons were performed, simple main effects were found for time for the FIELD groups $\left(F_{2,12}=7.50 ; \mathrm{p}=0.008 ; \eta_{\mathrm{p}}{ }^{2}=0.56\right)$ with post-hoc comparisons showing significant differences between BL and Trial4 $(p=0.03)$. No other differences were revealed.

For the TMT-A test, there were no significant main effects for time $\left(\mathrm{F}_{2,40}=1.88 ; \mathrm{p}=0.17 ; \eta_{\mathrm{p}}{ }^{2}\right.$ $=0.09$ ) or interaction effect within subjects $\left(\mathrm{F}_{4,40}=1.14 ; \mathrm{p}=0.35 ; \eta_{\mathrm{p}}{ }^{2}=0.10\right)$. However, there was a significant main effect between subjects $\left(\mathrm{F}_{2,20}=4.60 ; \mathrm{p}=0.02 ; \eta_{\mathrm{p}}{ }^{2}=0.31\right)$. Simple main effects were found for BL results $\left(\mathrm{F}_{2,20}=4.78 ; \mathrm{p}=0.02 ; \eta_{\mathrm{p}}{ }^{2}=0.31\right)$, with post-hoc comparisons showing the differences lay between the FIELD and LAB groups $(\mathrm{p}=$ 0.02 ). No other between groups differences were revealed. However, again when planned comparisons were run, simple main effects were found for time for the FIELD groups $\left(F_{2,12}=7.50 ; p=0.008 ; \eta_{p^{2}}=0.56\right)$ with post-hoc comparisons showing significant differences between BL and Trial4 $(p=0.03)$. Analysis of the TMT-B results revealed no significant main effect between subjects $\left(\mathrm{F}_{2,20}\right.$ $\left.=2.54 ; \mathrm{p}=0.10 ; \eta_{\mathrm{p}}{ }^{2}=0.20\right)$ or for the within subject interaction effect $\left(\mathrm{F}_{4,40}=1.68 ; \mathrm{p}=0.16\right.$; $\left.\eta_{\mathrm{p}}{ }^{2}=0.14\right)$. However, there was a significant within subject main effect for time $\left(\mathrm{F}_{2,40}=5.43\right.$; $\left.\mathrm{p}=0.008 ; \eta_{\mathrm{p}}{ }^{2}=0.21\right)$. A significant simple main effect for time was found for the $\mathrm{LAB}$ group $\left(\mathrm{F}_{2,14}=4.31 ; \mathrm{p}=0.04 ; \eta_{\mathrm{p}}{ }^{2}=0.38\right)$, however post-hoc comparisons did not reveal where these differences lay. Similarly, there was a significant simple main effect for time 
for the CONTROL group $\left(\mathrm{F}_{2,14}=13.70 ; \mathrm{p}=\right.$ $\left.0.001 ; \eta_{\mathrm{p}}{ }^{2}=0.66\right)$, whilst post-hoc comparisons showed differences lay between BL and Trial1 $(\mathrm{p}=0.01)$ and BL and Trial4 $(\mathrm{p}=0.02)$. findings indicate that Downhill Mountain biking does appear to impair executive function in this cohort.

Table 1. Mean and standard deviation for recorded variables at baseline (BL) and following Trial1 and Trial4. Data reported are the mean \pm SD.

\begin{tabular}{|c|c|c|c|}
\hline Test & FIELD & LAB & CONTROL \\
\hline \multicolumn{4}{|c|}{ Stroop Test OffTime (s) } \\
\hline $\mathrm{BL}$ & $47.08 \pm 5.18$ & $53.91 \pm 5.48$ & $53.19 \pm 7.97$ \\
\hline Trial1 & $55.22 \pm 6.49^{*}$ & $53.24 \pm 7.43$ & $53.90 \pm 8.02$ \\
\hline Trial4 & $53.38 \pm 5.14^{*}$ & $50.60 \pm 6.72^{*}$ & $53.44 \pm 7.55$ \\
\hline \multicolumn{4}{|c|}{ Stroop Test OnTime (s) } \\
\hline $\mathrm{BL}$ & $54.48 \pm 7.36$ & $58.37 \pm 7.66$ & $57.13 \pm 9.64$ \\
\hline Trial1 & $63.11 \pm 8.17^{*}$ & $55.06 \pm 7.61$ & $57.17 \pm 11.00$ \\
\hline Trial4 & $62.51 \pm 8.52^{*}$ & $56.10 \pm 7.78$ & $57.87 \pm 10.96$ \\
\hline \multicolumn{4}{|c|}{ Stroop Test OffTrials (No.) } \\
\hline $\mathrm{BL}$ & $5.14 \pm .38$ & $5.75 \pm .71$ & $5.63 \pm .74$ \\
\hline Trial1 & $5.14 \pm .38$ & $5.88 \pm 1.46$ & $5.38 \pm .52$ \\
\hline Trial4 & $5.86 \pm 1.07$ & $5.25 \pm .46$ & $5.87 \pm .64$ \\
\hline \multicolumn{4}{|c|}{ Stroop Test OnTrials (No.) } \\
\hline $\mathrm{BL}$ & $5.14 \pm .38$ & $5.50 \pm .76$ & $5.87 \pm .84$ \\
\hline Trial1 & $5.57 \pm .54$ & $6.00 \pm 1.07$ & $5.75 \pm 1.04$ \\
\hline Trial4 & $6.29 \pm .49^{*}$ & $6.00 \pm 1.20$ & $6.13 \pm .99$ \\
\hline \multicolumn{4}{|c|}{ Stroop Test OnTime-OffTime (s) } \\
\hline $\mathrm{BL}$ & $7.41 \pm 4.60$ & $4.46 \pm 4.80$ & $3.94 \pm 4.16$ \\
\hline Trial1 & $7.89 \pm 6.54$ & $1.82 \pm 3.42$ & $3.27 \pm 5.44$ \\
\hline Trial4 & $9.14 \pm 5.13^{*}$ & $5.50 \pm 5.62$ & $4.43 \pm 5.89$ \\
\hline \multicolumn{4}{|c|}{ Trail Making Test A (TMT-A) (s) } \\
\hline$\overline{\mathrm{BL}}$ & $13.94 \pm 2.24$ & $20.13 \pm 4.13^{+}$ & $17.86 \pm 4.69$ \\
\hline Trial1 & $12.48 \pm 2.65$ & $15.98 \pm 2.65$ & $17.19 \pm 4.93$ \\
\hline Trial4 & $11.29 \pm 1.87^{*}$ & $19.71 \pm 10.73$ & $15.75 \pm 4.73$ \\
\hline \multicolumn{4}{|c|}{ Trail Making Test B (TMT-B) (s) } \\
\hline BL & $39.62 \pm 9.19$ & $40.41 \pm 9.84$ & $31.59 \pm 8.14$ \\
\hline Trial1 & $38.91 \pm 12.82$ & $29.63 \pm 11.30$ & $27.24 \pm 7.85^{*}$ \\
\hline Trial4 & $38.71 \pm 12.80$ & $34.68 \pm 9.68$ & $27.30 \pm 8.28^{*}$ \\
\hline
\end{tabular}

Significance set at $\mathrm{p} \leq .05 .{ }^{*}$ indicates significantly different to $\mathrm{BL} ;{ }^{+}$indicates significantly different to FIELD; ${ }^{\mathrm{H}}$ indicates significantly different to LAB.

\section{Discussion}

The purpose of this study was to investigate the possible effect multiple short-duration DHI runs over a single day had on various measures of executive function when compared to comparably fatiguing repeated sprint exercise within a laboratory setting and a non-exercising control group. The key
Results of the Stroop test showed that OffTime was significantly slower following Trial1 and Trial4 $(14.75 \%$ and $11.81 \%$, respectively) when compared to baseline times for the FIELD group. Conversely, the LAB group were approximately $6 \%$ quicker following Trial4 compared to baseline, whilst the CONTROL showed no change across time points. The FIELD group also showed 
similar decrements in OnTime performance following Trial1 and Trial4 compared to $\mathrm{BL}$, whilst neither LAB or CONTROL groups showed any significant changes.

While there were differences in the gender composite of the LAB and CONTROL groups compared to the FIELD group it is unlikely that this would have biased the results toward positive findings. Previous research has reported the opposite, with women showing greater susceptibility to cognitive decline than males (Lynall et al, 2016). However, this was not shown to be the case in the present study.

As the EncephalApp Stroop test had practice trials built into it and all participants where given the opportunity to familiarise themselves with the app, the results indicate that performance in both basic psychomotor speed and executive functioning, such as inhibition, selective attention and shift ability, diminished following several downhill runs. Whilst previous research has shown an acute bout of exercise can facilitate improvements in cognition (Sibley, Etnier \& Le Masurier, 2006), as observed in the LAB group, this doesn't explain the significant reductions in cognitive performance observed in the FIELD group. Therefore, these negative changes may be the result of the repeated head accelerations the FIELD group were exposed to due to the terrain ridden over, thus negating any potential cognitive benefits from the exercise itself.

Results of the present study may be comparable to data on repeated heading of footballs and the negative relationship between the number of headers made and cognitive impairments to executive functioning (Matser, Kessels, Lezak \& Troost, 2001). As DHI also involves repeated head accelerations, typically of a greater mean magnitude to those seen in soccer (Lynall et al., 2016; Hurst, Atkins \& Dickinson, 2018), it is plausible that these contributed to the decline in executive function observed in the present study. Indeed, estimates of effect size for OffTime and OnTime were classified as large (0.69 and 0.42, respectively) for the FIELD group, indicating that $69 \%$ and $42 \%$ of the variance in the scores were likely attributable to performing multiple DHI runs and potentially the influence of head accelerations.

It should also be acknowledged that equipment used may also influence the magnitude of head accelerations. Whilst all riders in the FIELD group used the same team issue bicycles with identical componentry, the front and rear suspension was set up to the riders' personal preferences. Subsequently, how soft or hard the shocks were and how much compression and rebound dampening was set might have affected how much trail shock was transmitted to the head. Despite this, Hurst, Atkins and Dickinson (2018) reported all DHI riders in their study, irrespective of bicycle design and set up, still experienced numerous linear and rotational head acceleration above approximately $80 \mathrm{~g}$ and $8500 \mathrm{rad} / \mathrm{s}^{2}$, respectively. Therefore, it is questionable as to how effective different bicycle set ups are in attenuating these high transmitted forces to the head.

While there were no significant differences in the number of trials required to complete the easier congruent "Off" state, either between groups or within groups at each time point, the FIELD group did requiring significantly more attempts to complete five successful incongruent "On" state trials without error, following Trial4 compared to BL. This suggests that processing accuracy decreased following several DHI runs, and again, may be attributed to the repeated translational and rotational accelerations previously identified within this sport (Hurst, Atkins \& Dickinson, 2018).

Cognitive flexibility, as determined by the OnTime-OffTime score, was significantly slower from BL to Trial4 for the FIELD group (18.93\%). However, while not quite reaching a level of significance, the LAB group showed almost identical decrements (18.91\%), while the CONTROL group showed an increase of $11.07 \%$ from BL to Trial4. This may indicate an element of fatigue in both exercise groups influenced cognitive flexibility over the course of the trials, whilst a degree of learning may have occurred in the control group. 
Previous research has indicated a doseresponse to exercise intensity and cognitive function, with performance improving with higher intensities (Chang \& Etnier, 2009). However, despite the high intensity nature of DHI, the results of the present study do not support Chang and Etnier (2009). This is again potentially due to the repeated head acceleration experienced by DHI riders. As previously alluded to, repeated subconcussive head accelerations have the capacity to cause cerebral or neurological inhibition, and therefore have the potential to impair cognitive function (Broglio et al., 2011; McAllister et al., 2014; Alosco et al., 2018), again overriding any potential benefits conferred by high intensity exercise.

Results of the TMT-A found a significant difference between $\mathrm{BL}$ and Trial4 for the FIELD group, with time to complete the task improving $\sim 19 \%$. However, neither the LAB nor CONTROL groups showed any significant differences in TMT-A times. For the harder TMT-B test, both LAB and CONTROL groups were significantly quicker following each trial compared to BL, whilst the FIELD group showed no change. These results may indicate an improvement in behavioral regulation and motor speed in the FIELD group due to simple reaction time improving over the course of the day as a consequence of riding the same course. As such, this may have helped to improve this component of cognitive function. However, as a result of performing DHI, more complex tasks, as indicated by the TMT-B test, were inhibited or at least unhanged when compared to the LAB and CONTROL groups, again potentially due to repeated head accelerations.

\section{Practical Applications.}

This study shows that repetitive, short-term oscillation from Downhill mountain biking, at least temporarily, significantly impairs measures of executive function following a single day of riding in the cohort tested. Therefore, riders and coaches should be mindful that Downhill mountain biking might be contraindicated for mental health, despite the known cardio-respiratory benefits. Given the repeated sub-concussive head accelerations in DHI and the negative effects these can have on cognitive function, similar to those observed in field-based collision sports, it is suggested that mountain biking also be investigated as a collision sport and future research should focus on trying to reduce these accelerations to minimise potential cognitive decline.

\section{Conclusions}

These results indicate that just four DHI runs were sufficient to impair measures of executive functioning when compared to performing comparably fatiguing repeated sprints without cerebral accelerations within a laboratory setting. Given that a typical race weekend may involve upwards of 12 downhill runs and that the competition season involves more than a dozen races, these results suggest that riders participating in DHI may be at an increased risk of sustaining cognitive impairments, comparable to studies of team sport athletes after a season of play (McAllister et al., 2014). As such, downhill mountain biking might be contraindicated for mental health, despite its known cardio-respiratory benefits.

Given that the degenerative disease CTE appears to have a dose response of about 4.5+ years of playing a sport with repeated subconcussive impacts (Alosco et al., 2018), and that our FIELD participants had a minimum of 4 years of riding experience, results from this study suggest that long-term cognitive degeneration should be examined for in deceased mountain bike riders, too. Whilst the authors accept that sample size was low, those in the FIELD group did represent $~ 12 \%$ of all UK registered Elite DHI riders for the 2018 season and therefore provided a representative sample. However, these preliminary findings should be viewed as indicative rather than definitive and thus further longitudinal studies are warranted on larger cohorts and of riders of varying age and ability levels to establish any such longterm effects of executive functioning. 
Funding: This research received no external funding.

Acknowledgments: We would like to acknowledge all the participants who took part in this study and thank them for their kind cooperation and giving up their time. This research did not receive any specific grant from funding agencies in the public, commercial, or not-for-profit sectors.

Conflicts of Interest: The authors declare no conflict of interest.

\section{References}

1. Alosco ML, Mez J, Tripodis Y, et al. Age of first exposure to tackle football and chronic traumatic encephalopathy. Ann Neurol 2018; 83(5): 886-901.

2. Bajaj JS, Heuman DM, Sterling $R$, et al. Validation of EnceplalApp, smartphonebased Stroop test for the diagnosis of covert hepatic encephalopathy. Clin Exp Gastroenterol Hepatol 2015; 13(10): 18281825.

3. Becker J, Runer A, Neunhäuserer D, et al. Prospective study of downhill mountain biking injuries. Br J Sports Med 2013; 47(7): 458-462.

4. Broglio SP, Eckner JT, Martini D, et al. Cumulative head impact burden in high school football. J Neurotrauma 2011; 28(10): 2069-2078.

5. Broglio SP, Schnebel B, Sosnoff JJ, et al. The biomechanical properties of concussions in high school football. Med Sci Sports Exerc 2010; 42(11): 2064-2071.

6. Burr JF, Drury $\mathrm{CT}$, Ivey $\mathrm{AC}$, et al. Physiological demands of downhill mountain biking. J Sports Sci 2012; 30(6): 1777-1785.

7. Chang YK, Etnier JL. Exploring the doseresponse relationship between resistance exercise intensity and cognitive function. J Sport Exerc Psychol 2009; 31(5): 640-656.

8. Cohen J. Statistical power analysis for behavioural sciences (2nd Ed.). Hilldale, NJ: Erlbaum, 1988.

9. Di Virgilio TG, Hunter A, Wilson L, et al. Evidence for acute electrophysiological and cognitive changes following routine soccer heading. EBioMedicine, 2016; 13: 66-71.
10. Donaldson L, Asbridge M, Cusimano MD. Bodychecking rules and concussion in elite hockey. PloS one 2013; 8(7): e69122.

11. Gardner A, Iverson GL, Levi CR, et al. A systematic review of concussion in rugby league. Br J Sports Med 2015; 49(8): 495-498.

12. Guskiewicz KM, McCrea M, Marshall SW, et al. Cumulative effects associated with recurrent concussion in collegiate football players: the NCAA Concussion Study. Jama 2003; 290(19): 2549-2555.

13. Guskiewicz KM, Mihalik JP, Shankar V, et al. Measurement of head impacts in collegiate football players: relationship between head impact biomechanics and acute clinical outcome after concussion. Neurosurg 2007; 61(6): 1244-1253

14. Hurst HT, Atkins S, Dickinson BD. The magnitude of translational and rotational head accelerations experienced by riders during downhill mountain biking. J Sci Med Sport 2018; 21(12): 1256-1261.

15. Hurst HT, Atkins S. Agreement between polar and SRM mobile ergometer systems during laboratory-based high-intensity, intermittent cycling activity. J Sports Sci 2006; 24(8): 863-868.

16. Hurst HT, Rylands L, Atkins S, et al. Profiling of translational and rotational head accelerations in youth BMX with and without neck brace. J Sci Med Sport 2018; 21(3): 263267.

17. Kronisch RL, Pfeiffer RP, Chow TK. Acute injuries in cross-country and downhill offroad bicycle racing. Med Sci Sports Exerc 1996; 28(11): 1351-1355.

18. Kroshus E, Garnett B, Hawrilenko M, et al. Concussion under-reporting and pressure from coaches, teammates, fans, and parents. Social science \& medicine 2015; 134: 66-75.

19. Lynall RC, Clark MD, Grand EE, et al. Head impact biomechanics in women's college soccer. Med Sci Sports Exerc 2016; 48(9): 17721778.

20. Maroon JC, Winkelman R, Bost J, et al. Chronic traumatic encephalopathy in contact sports: a systematic review of all reported pathological cases. PloS one 2015; 10(2): e0117338.

21. Matser JT, Kessels AG, Lezak MD, Troost J. A dose-response relation of headers and concussions with cognitive impairment in professional soccer players. J Clin Exp Neuropsychol 2001; 23(6): 770-774. 
22. McAllister TW, Ford JC, Flashman LA, et al. Effect of head impacts on diffusivity measures in a cohort of collegiate contact sport athletes. Neurology 2014; 82(1): 63-69.

23. McGehee DV, Carsten OMJ. Perception and biodynamics in unalerted precrash response. Ann Adv Automot Med 2010; 54: 315-332.

24. Meaney DF, Smith DH. Biomechanics of concussion. Clin Sports Med 2011; 30(1): 1931.

25. Mez J, Daneshvar DH, Abdolmohammadi B, et al. Duration of American football play and chronic traumatic encephalopathy. Ann Neurol 2020; 87(1): 116-131.

26. Reitan RM. Validity of the Trail Making Test as an indicator of organic brain damage. Percept Mot Skills 1958; 8(3): 271-276.

27. Russell ER, Stewart K, Mackay DF, et al. Football's Influence on Lifelong health and
Dementia risk (FIELD): protocol for a retrospective cohort study of former professional footballers. BMJ open 2019; 9: e028654.

28. Sibley BA, Etnier JL, Le Masurier GC. Effects of an acute bout of exercise on cognitive aspects of Stroop performance. J Sport Exerc Psychol 2006; 28(3): 285-299.

29. Spiotta AM, Shin JH, Bartsch AJ, et al. Subconcussive impact in sports: a new era of awareness. World Neurosurg 2011; 75(2):175178.

30. Stern RA, Riley DO, Daneshvar DH, et al. Long-term consequences of repetitive brain trauma: chronic traumatic encephalopathy. Pm\&r 2011; 3(10): S460-467.

31. Thompson MD, Scott JG, Dickson SW, et al. Clinical utility of the Trail Making Test practice time Clin Neuropsychol 1999; 13(4):450-455. 